\title{
Stellar population history of Seyfert 2 nuclei
}

\author{
R. Rodrigues Lacerda ${ }^{1}$, R. Cid Fernades ${ }^{1}$, Q. Gu ${ }^{2}$, J. Melnick ${ }^{3}$, \\ E. Terlevich ${ }^{4}$, R. Terlevich ${ }^{4,5}$ and D. Kunth ${ }^{6}$ \\ ${ }^{1}$ Universidade Federal de Santa Catarina, Florianópolis-SC, Brazil \\ ${ }^{2}$ Department of Astronomy, Nanjing University, Nanjing 210093, P. R. China \\ ${ }^{3}$ European Southern Observatory, Alonso de Codorva 3107, Santiago, Chile \\ ${ }^{4}$ INAOE, Tonantzintla, Puebla, Mexico \\ ${ }^{5}$ Institute of Astronomy, Madingley Road, CB3 OHA Cambridge, UK \\ ${ }^{6}$ Institut d'Astrophysique de Paris, 98bis Bouleward Arago, 75014 Paris, France
}

\begin{abstract}
We present the results of a new stellar population synthesis code which mixes both evolutionary and empirical population synthesis techniques. The code decomposes a given galaxy spectrum into a base of high resolution spectra from the recent evolutionary synthesis models of Bruzual \& Charlot (2003). The main novelty is that now we synthesize the whole $F_{\lambda}$ spectrum, rather than a handful of absorption lines and colors.
\end{abstract}

\section{The data set}

The dataset used in this contribution consist of 79 nearby galaxies from the Atlas of Joguet et al. (2001). The sample comprises 65 type 2 Seyferts, plus some LINERs, Seyfert 1s, Starburst and normal galaxies. The spectra cover the wavelength range $3500-5500 \AA$.

\section{The method}

The synthesis code searches for the linear combination of N Simple Stellar Populations of same age $(t)$ and metallicity $(Z)$, plus a $\nu^{1.5}$ Featureless Continuum (FC), which best matches a given observed spectrum. It outputs a population vector $\vec{x}$ which carries the flux fractions associated to each population $(t i, Z i)$ in the base.

The new code also provides the measurement of velocity dispersion, which we find to be consistent with values from literature. In this contribution we present a first application of this method for a large sample of Seyfert 2s. Figure 1 shows the an example of the spectral fits.

\section{Main results}

(i) Our new population synthesis code, built upon the new models by Bruzual \& Charlot, is capable of reproducing observed spectra to within the noise level. The star formation history deduced from the fits are robust as long as one does not attempt an overdetailed description.

(ii) The stellar population history of Seyfert $2 \mathrm{~s}$ is remarkably varied. Old stars, intermediate age populations and young starbursts all appear in significant proportions.

(iii) The starlight-subtracted spectra were used to investigate the presence of weak broad emission features which are hard to detect in the total spectrum. This analysis 

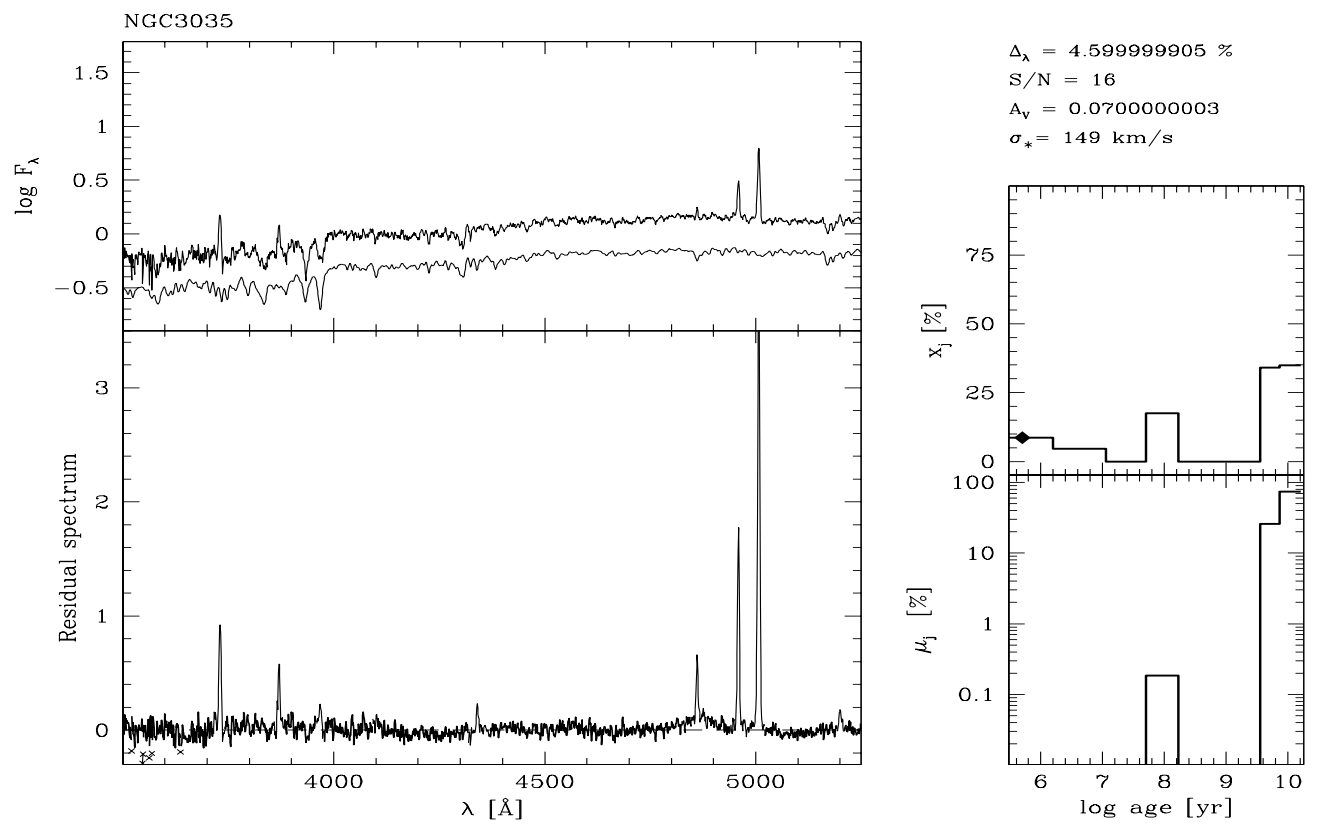

Figure 1. Examples of the spectral fits. The top left panel shows the logarithm of the observed and the synthetic spectra. The residual spectrum is shown in the bottom left. Spectral regions actually used in the synthesis are plotted with a black line and masked regions are plotted in blue. The top-right panel shows the population vector $\vec{x}$, i.e., the flux fraction (at $\lambda_{\mathrm{o}}=4020 \AA$ ) associated with populations of a given age. The corresponding mass fractions vector $\vec{m}$ is shown in the bottom.

revealed the signatures of WR stars in 3 (maybe 5) Seyfert 2s, all of which have large young stellar population as deduced by the synthesis.

(iv) The analysis of the "pure-emission" spectra further allowed the detection of a weak BLR-like component under $H_{\beta}$ in several Seyfert 2s. For most of these objects independent spectropolarimetry data reveals a type 1 spectrum, and our synthesis indicate significant FC fractions. We are thus detecting in direct, non-polarized light the scattered BLR and AGN continuum predicted by the unified model! (Cid Fernandes \& Terlevich 1995).

\section{Acknowledgements}

Special thanks to CNPq and PRONEX by financial support.

\section{References}

Bruzual G., \& Charlot S. 2003, MNRAS, 344, 1000

Cid Fernandes R., \& Terlevich R. 1995, MNRAS, 272, 423

Joguet B., Kunth D., Melnick J., \& Terlevich R. 2001, A\&A, 380, 19 